\section{RÁPIDA PROGRESIÓN DE LA ENFERMEDAD RENAL CRÓNICA EN POBLACIÓN PERUANA - RÉPLICA}

\section{RAPID PROGRESSION OF CHRONIC KIDNEY DISEASE IN THE PERUVIAN POPULATION - REPLY}

\author{
Jessica Bravo-Zúñiga ${ }^{1, a}$, Ricardo Chávez-Gómez ${ }^{1, a}$, \\ Jungmei Gálvez-Inga ${ }^{1, a}$, Mirko Villavicencio-Carranza1,a, \\ José Espejo-Sotelo ${ }^{1, a}$, Manuel Riveros-Aguilar ${ }^{1, a}$
}

Sr. Editor. Nos alegra el interés que ha despertado nuestra publicación previa, pues nos permite discutir el problema y hacer las siguientes precisiones:

La progresión y la evolución de la enfermedad renal crónica (ERC) es muy variable entre los sujetos que la padecen. Cuando no se dispone de evidencias suficientes para definir e identificar a aquellos que tendrán una progresión rápida, se recomienda evaluar simultánea y sistemáticamente la tasa de filtración glomerular estimada (TFGe) y la albuminuria ${ }^{(1)}$.

La Guía de la Kidney Disease Improving Global Outcomes: KDIGO, define la progresión de la ERC como un descenso sostenido del filtrado glomerular (FG) $>5 \mathrm{~mL} / \mathrm{min} / 1,73 \mathrm{~m}^{2}$ al año o por el cambio de categoría, siempre que este se acompañe de una pérdida de $\mathrm{FG} \geq 5 \mathrm{~mL} / \mathrm{min} / 1,73 \mathrm{~m}^{2}$ (2).

El grado de precisión con que la progresión es capaz de ser estimado, depende, en gran medida, de dos factores: número de mediciones de creatinina sérica utilizadas para definir la progresión y la duración del seguimiento.

En nuestro estudio, la mediana de seguimiento fue 12,4 meses (Rango Intercuartilico: 5,1-27,2) y la forma de calcular la progresión fue mediante la diferencia entre dos valores de TFGe, dividido entre el tiempo de seguimiento individual. Ciertamente, los pacientes que progresaron

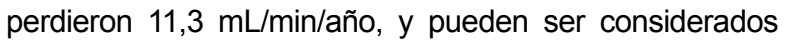
progresores rápidos, a pesar de que entre los evaluados no hubo ningún paciente con diabetes tipo 1, por tanto, un sesgo puede ser el hecho de haber tomado solo dos puntos de corte.

\footnotetext{
Unidad de Salud Renal, Departamento de Nefrología, Hospital Nacional Edgardo Rebagliati Martins. Lima, Perú

a Médico nefrólogo

Recibido: 03/09/2017 Aprobado: 20/09/2017 En línea: 20/09/2017
}

Citar como: Bravo-Zúñiga J, Chávez-Gómez R, Gálvez-Inga J, VillavicencioCarranza M, Espejo-Sotelo J, Riveros-Aguilar M. Rápida progresión de la enfermedad renal crónica en población peruana - réplica. Rev Peru Med Exp Salud Publica. 2017;34(3):570-1. doi: 10.17843/rpmesp.2017.343.3098
Tabla 1. Progresión de ERC según etiología de enfermedad y nivel de proteinuria $>1,5 \mathrm{~g} /$ día

\begin{tabular}{lccc}
\hline $\begin{array}{l}\text { Pacientes que } \\
\text { progresaron }\end{array}$ & \multicolumn{2}{c}{ Proteinuria $>\mathbf{1 , 5}$ g/día } & \multirow{2}{*}{ Total } \\
\cline { 2 - 3 } $\begin{array}{l}\text { según etiología de } \\
\text { enfermedad }\end{array}$ & $\begin{array}{c}\text { Sí } \\
\mathbf{n}(\%)\end{array}$ & $\begin{array}{c}\text { No } \\
\mathbf{n}(\%)\end{array}$ & \\
\hline Diabetes mellitus & $30(52,6)$ & $27(47,4)$ & 57 \\
\hline Hipertensión arterial & $38(13,6)$ & $242(86,4)$ & 280 \\
\hline $\begin{array}{l}\text { Glomerulonefritis } \\
\text { crónica }\end{array}$ & $6(60,0)$ & $4(40,0)$ & 10 \\
\hline Uropatía obstructiva & $1(20,0)$ & $4(80,0)$ & 5 \\
\hline
\end{tabular}

Sobre el tipo de población, efectivamente, el mayor grupo corresponde a hipertensos con 1026 (82,2\%); de ellos, 280 $(27,3 \%)$ progresaron en su enfermedad renal, y el $13,6 \%$ de estos presentó proteinuria $>1,5 \mathrm{~g} / \mathrm{d}$; sin embargo, fueron los pacientes con diabetes mellitus y glomerulopatía crónica los que presentaron con más frecuencia esta característica, aunque el número total de casos de pacientes con esta etiología fue mucho menor (Tabla 1).

Al respecto, es cierto que ante un paciente hipertenso de larga evolución se considera clásicamente la presencia de proteinuria inferior a $1 \mathrm{~g} / \mathrm{d}$ y una lenta progresión de la insuficiencia renal, y se asume que, probablemente, se trate de una nefroangioesclerosis hipertensiva $(\mathrm{NH})$, sin realizar una biopsia renal. Sin embargo, existen reportes como el de Martin Alemany et al. (3) donde se comparan las características clínicas y evolutivas de pacientes afectados de $\mathrm{NH}$ con proteinuria en rango nefrótico, con otras enfermedades glomerulares, encontrando que estos pacientes eran de mayor edad, mayor tiempo de evolución, recibían varios fármacos hipotensores, presentaban albumina normal y ausencia de edemas, y una TFG menor al momento de la biopsia renal $\left(32 \mathrm{~mL} / \mathrm{min} / 1,73 \mathrm{~m}^{2}\right)$.

Por tanto, no puede considerarse excepcional y, de hecho, corresponde al $5 \%$ de las biopsias renales reportadas, los pacientes en quienes se asocia $\mathrm{NH}$ y proteinuria $>1 \mathrm{~g} /$ día asociándose a un pobre pronóstico renal ${ }^{(4,5)}$.

Finalmente, respecto a los pacientes que podrían desarrollar insuficiencia renal aguda, en la sección de materiales y métodos se indica que se excluyeron los pacientes con deterioro de la función renal en los tres meses previos, debido a nefrotoxicidad, deshidratación, infección o cirugía.

Contribución de los autores: todos hicieron la revisión de la bibliografía y redacción del manuscrito.

Fuentes de financiamiento: autofinanciado.

Conflictos de interés: los autores declaramos no tener conflictos de interés. 


\section{REFERENCIAS BIBLIOGRÁFICAS}

1. Gorostidi M, Santamaría R, Alcázar R, Fernández-Fresnedo $\mathrm{R}$, Galceran JM, et al. Documento de la Sociedad Española de Nefrología sobre las guías KDIGO para la evaluación y el tratamiento de la enfermedad renal crónica. Nefrologia. 2014;34(3):302-16. doi:10.3265/Nefrologia.pre2014.Feb.12464.

2. Chapter 2: Definition, identification, and prediction of $\mathrm{CKD}$ progression. Kidney Int Suppl (2011). 2013;3(1):63-72. doi: 10.1038/kisup.2012.65

3. Martín Alemany, N, Almirall Daly J, Orellana Fernández R, Andreu X. Proteinuria de tipo nefrótico en la nefroangioesclerosis hipertensiva: características clínicas y evolutivas. Med Clin (Barc). 2014;143(9):392-4. doi: 10.1016/j.medcli.2013.09.015.

4. Torres-Conde S, Herrador-Paredes J, Muñoz-Abad L, LozanoFernández MJ, Pozuelos-Estrada J. Nefroangioesclerosis hipertensiva, a propósito de un caso con proteinuria en rango nefrótico, sin hipoalbuminemia ni edemas. Semergen. 2015;41(8):e75-7. doi: 10.1016/j.semerg.2014.10.001

5. Obialo CI, Hewan-Lowe K, Fulong B. Nephrotic proteinuria as a result of essential hypertension. Kidney Blood Press Res. 2002;25(4):250-4.

Correspondencia: Jessica Bravo Zuñiga

Dirección: Francisco Graña 674, dpto 204. Lima 17, Perú

Teléfono: (+511) 995020905

Correoelectrónico:ivobz@yahoo.es

\section{ETIQUETADO DE ALIMENTOS Y PROCESADOS: UNA HERRAMIENTA NECESARIA PARA LA EDUCACIÓN ALIMENTARIA}

\section{FOOD AND PROCESSED FOOD LABELLING: AN INSTRUMENT NEEDED FOR ALIMENTARY EDUCATION}

Helard Manrique ${ }^{1,3, a}$, Miguel Pinto ${ }^{2, a}$, Vanessa Sifuentes ${ }^{3, b}$

Sr. editor. Hemos leído el Decreto Supremo (DS) 017 2017-SA, publicado el día 17/6/17 (1), que sustituye al D.S. 007-2015-SA del 18/4/15. Este DS establece nuevos

\footnotetext{
Servicio de Endocrinología, Hospital Nacional Arzobispo Loayza. Lima, Perú. Servicio de Endocrinología, Hospital Nacional Cayetano Heredia. Lima, Perú.

3 Centro de Investigación en Diabetes Obesidad y Nutrición. Lima, Perú. a Médico endocrinólogo, ${ }^{\mathrm{b}}$ nutricionista.

Recibido: 24/07/2017 Aprobado: 09/08/2017 En línea: 29/09/2017
}

Citar como: Manrique H, Pinto M, Sifuentes V. Etiquetado de alimentos y procesados: una herramienta necesarioa para la educación alimentaria. Rev Peru Med Exp Salud Publica. 2017;34(3):571-2. doi: 10.17843/rpmesp.2017.343.3020 parámetros técnicos. Llama la atención que, a los seis meses de su aprobación, se permitirá hasta $6 \mathrm{~g}$ de azúcar/100 mL para bebidas y hasta $22,5 \mathrm{~g}$ de azúcar/100 $\mathrm{g}$ para alimentos sólidos. Recién al sobrepasar dichas cantidades el alimento deberá llevar una advertencia que indique "Alto en".

EI DS también establece que, a los 39 meses de publicado -periodo que nos parece excesivo-, las empresas deberán ajustarse a nuevos límites (hasta $5 \mathrm{~g}$ de azúcar/100 mL en bebidas y hasta $10 \mathrm{~g} / 100 \mathrm{~g}$ en alimentos sólidos) (1); exactamente el doble de lo que permitía el reglamento del 2015. Estudios recientes de prevalencia de sobrepeso y obesidad muestran que nuestro país está inmerso en una epidemia, ya que el $53,8 \%$ de los peruanos tiene sobrepeso y obesidad (2), debido a que vivimos en un ambiente de alimentos hipercalóricos en donde, culturalmente, predominan los regímenes alimentarios excesivos, mal equilibrados y, por ende, poco saludables.

El Perú carece aún de una política de salud pública para hacer frente a dicha pandemia, sin olvidar que el $7 \%$ de la población peruana tiene diabetes ${ }^{(3)}$.

El etiquetado de los alimentos vigente nos provee información de las cantidades de nutrientes que contienen; sin embargo, la mayoría de ciudadanos no logran comprenderla en su totalidad debido a su complejidad. En el Perú, un $5,7 \%$ de la población es analfabeta ${ }^{(4)}$ entonces, ¿cómo podemos pedirles que comprendan las etiquetas de los productos que consumen?

En el Ecuador, la experiencia del "semáforo nutricional (SN)" está vigente desde el 2014. Ellos consideran que un producto tiene bajo contenido de azúcar cuando contiene $2,5 \mathrm{~g} / 100 \mathrm{~mL}$ de producto líquido y $5 \mathrm{~g} / 100 \mathrm{~g}$ de producto sólido ${ }^{(5)}$. Si el producto cumple con este requerimiento, entonces, delante de su envoltura se colocará un círculo verde y dentro de él estará escrito "bajo en azúcar". La Organización Mundial de Salud apoya esta norma desde sus lineamientos de alimentación saludable. Estudios han demostrado que el SN ha tenido un efecto positivo en el conocimiento y comprensión del contenido de los productos procesados. Las prácticas relacionadas a la compra y consumo de alimentos procesados mejoran a través de la promoción de la política del etiquetado nutricional, control de la comercialización, y el monitoreo y la vigilancia de su implementación ${ }^{(6)}$.

Esto no solo busca que los ciudadanos puedan tener una elección más consiente, sino también, un incentivo para que las industrias reformulen la cantidad de grasas, azúcar y sal que colocan en sus preparaciones. 